\title{
Application of Vacuum Membrane Distillation in Water Treatment
}

\author{
Yajing Li \& Kunpeng Tian \\ School of science, Tianjin Polytechnic University, Tianjin 300160, China \\ E-mail: duck10625@yahoo.com.cn
}

\begin{abstract}
In the present paper, we reviewed the principles and classification of membrane distillation, with an emphasis on the factors affecting it and approaches to enhance its efficacy, and finally outlook its research prospects.
\end{abstract}

Keywords: Vacuum membrane distillation, Classification, Affecting factors, Research prospects

\section{Introduction}

Water is the source of life, the basis of human survival, and the principal material base to guarantee the economy substantial development of a country. However, a practical and urgent strategic problem emerged in the world, viz. Water resource crisis. In the early $20^{\text {th }}$ century, there was the parlance in the world that "Struggling for coal in $19^{\text {th }}$ century, oil in $20^{\text {th }}$ century and water in $21^{\text {th }}$ century" $(\mathrm{Li}, 2001$, PP. 166-167). However, $2 / 3$ surface of the earth is covered by water, and solutions to water resource crisis is actually how to utilize water resource. As is well known to us all, $97.5 \%$ total water reserves in the globe is salt water, and among $2.5 \%$ fresh water, $2 \%$ water is tough to utilize for it is covered by icecaps, glaciers and ice-snow in two poles. The fresh water that could be utilized by human being in fact accounts for only $0.5 \%$ of total water reserves in the globe, while a majority of them belong to exhausted ground water which could not be renewable(Lin, 2000, PP. 7-12). Compared to the early $20^{\text {th }}$ century, the consumption of fresh water increased 6-7 times, and was two times of population increment. UN(United nation) have warned several times that almost $1 / 3$ population of the world would face the problem of water deficit in 2025 if without any powerful measurements in each country. China is listed in the table of 13 countries under the most severe water deficit. Gross fresh water in China make the top 6 in the world, but fresh water per capita is only $1 / 4$ of the average of the world, ranking 109 in the world. Moreover, temporal and spatial distribution of water is rather uneven(Bart, 2002, PP. 207-218). Accordingly, solutions to fresh water resources are urgent in order to guarantee the economy substantial development in China. Efficacious and strategic approach to settle fresh water resource crisis is the distillation of water.

Membrane distillation(MD) is a new membrane separation technology(Lawson, 1997, PP. 1-25; Schneider, 1986, PP. 514-521; Drioli, 1985, PP. 339-346), although it has been developed for only 10 years, due to its particular separation effects and promising application prospects, it have been paid more and more attention. Meanwhile, along with the progressing claims of environment protection recently, requirements for environment protection became higher and higher, and new technology is essential and urgent. Membrane distillation showed huge potential values in this area. Vacuum membrane distillation(VMD), as the emphasis and focus of MD, showed lower energy consumption and higher efficacy, and could be called as apotheosis of drag reducing and energy saving.

\section{Basic principles of MD}

MD was a membrane separation process combining with membrane technology and evaporation, and the membrane used was hydrophobic microporous membrane which could not be wetted by pending solutions. One side of membrane exposed to warm pending solutions directly(hot procedure side), the other side exposed to cold water solutions directly or indirectly(cold procedure side). Volatile components in hot procedure side evaporated in membrane surface, enter cold procedure side through membrane, and condensed into liquid phrase. Other components block off in hot procedure side by hydrophobic membrane and thus realize the objective of compounds separation and purification. MD was the process that transferred heat and quality simultaneously, and mass transfer impetus was steam differential imposed by components permeating through the both membrane sides.

\section{Classification of MD}

\subsection{Direct contact membrane distillation(DCMD)}

Both membrane sides contact with hot and cold water directly, respectively. It was suitable for water infiltration, such as desalination or condense water solutions. DCMD has successfully been applied in the wastewater treatment, and resulted in some infiltrations with low pollution to environment, such as treating textile wastewaters, pharmacy 
wastewater containing taurine, water with heavy metal ions and so on. DCMD could be also used to separate heat-sensitive materials, such as the condensation of juice, blood and so on(Chen, 1996, PP. 307-313).

\subsection{Air gap membrane distillation(AGMD)}

There was an air gap between cold procedure side and condensation wall, which could enhance the heat conduction drag. Therefore, flux of AGMD was usually smaller than that of other MDs, but AGMD had a broader application than DCMD, for AGMD condensed infiltration in the condensation surface, not directly. AGMD has been successfully applied in the production of pure water and the concentration of all kinds of nonvolatile solutes.

\subsection{Sweep gas membrane distillation(SGMD)}

Cold procedure side of membrane swept air in order to carry away the transferred steam. It used membrane to separate air or steam, in combination with lower heat conduction loss and lower mass transfer resistance. Infiltration condensed in the outer condenser. The charge of condenser was quite large, for in the large quantity of sweeping gas, only less vaporized filtration existed. Therefore, studies on SGMD were less available.

\subsection{Vacuum membrane distillation(VMD)}

Steam was continuously exerted from the cold procedure side of membrane by vacuum system, and condensed outside of the membrane separator. Porous membrane used in VMD served as the support of gas-liquid surface. This membrane possessed some selectivity, based on Knudsen diffusion rate of diffusing substances, but separation extent was mainly determined by the gas-liquid equilibrium condition in the membrane-solution surface. Compared to other MDs, one of the advantages was that heat conduction loss by membrane could be neglected. As a new separation approach, it was mainly applied to eliminate the volatile components in dilute aqueous solutions(Liu, 1991, P. 25) with larger membrane flux and greater industrial practical significance.

\section{Affecting factors during the process of VMD}

\subsection{Rejection rate}

Rejection rate was separation performance parameter of nonvolatile solute water solutions. Due to hydrophobicity of distillation membrane, its rejection rate was higher than that of other membrane separation(Xu, 1998, PP. 1-6). Its affecting factors are mainly two as follows: one is pore size, and $0.2-1.0 \mu \mathrm{m}$ pore size is usually considered to be proper, and $0.2-0.4 \mu \mathrm{m}$ is used much. Two is that pressure difference of membrane two sides could not exceed the pressure $\mathrm{P}^{*}\left(\mathrm{P}^{*}=2 \gamma \cos \theta \mathrm{R}\right)$ when liquids entered into membrane pore. Furthermore, rejection rate was related to solute concentration in the feeding system, feed flow rate and so on.

\subsection{Membrane permeation flux}

Membrane distillation has higher rejection rate but relatively lower flux, and flux affecting factors were outlined as follows(Zhang, 2001, PP. 16-21):

(1)Temperature: temperature was the main factor affecting permeation flux. For DCMD, enhancing the temperature of solutions in hot procedure side or temperature difference between two sides, could result in significant increment of water flux, but not in a linear relationship.

(2)Vapour pressure deficit: permeation flux increased with the increasing of vapour pressure deficit between both membrane sides, and a linear relationship was obtained.

(3)Feed concentration: with the increasing of feed concentration, flux of nonvolatile solute water solutions decreased, while that of volatile increased.

(4)Feed flow rate: Increasing feed flow or cooling water flow could result in flux increment.

(5)Distillation time: due to the process of distillation, membrane pore infiltration resulted in the reflux from the permeation side stream to feed, and membrane pollution caused the reduction of flux. Therefore, with the increasing of distillation time, flux reduction occurred.

(6)Membrane material and structure: hydrophobic microporous membrane was used in MD, and structure parameters affecting water flux were mainly as follows: pore size, porosity and membrane thickness.

\subsection{Thermal efficiency}

MD accompanied phase transition with heat energy consumption, and thermal efficiency affected directly its practical application. Thermal efficiency was an important factor which determined whether MD was competitive. Currently, thermal efficiency of MD was rather low( $30 \%$ or so), which was one of the key problems hindering its large scale industrial application. If pore size and porosity was larger, diffusion coefficient of steam in membrane pore was higher, and membrane thermal conductivity was lower. Variation of membrane thickness would affect the temperature of membrane surface, and thus have an indirect impact on evaporation efficiency. Taken together, thermal efficiency was close associated with membrane parameters, and therefore, it could be enhanced by ameliorating membrane parameters. 


\section{Approaches to enhance VMD performance}

\subsection{Attenuate concentration and temperature polarization}

During the process of VMD, in the interface of gas-liquid, temperature and concentration of solutions were all lower than main feed solutions, which was the concentration and temperature polarization during the process of VMD. These two polarizations would all resulted in the reduction of steam differential in both membrane sides, and thus caused the decline of permeation flux. According to MD mass transfer mechanisms, it would be beneficial to enhance MD flux by changing the flow state of feed solutions and attenuating concentration and temperature polarization. Bandini $\mathrm{S}$ et al(Bandini, 1999, PP. 58-62) augmented turbulent extent of thermal fluid by increasing feed flow, decreased the thickness of laminar boundary layer, and thus attenuated the concentration and temperature polarization of boundary layer, increased diffusion coefficient of thermal fluid and enhanced the mass transfer and heat transfer during the process. Martinez et al (Martinez, 1998, PP. 45-56) had placed spacers in the channel of feed solutions, and distillation flux increased $31 \% \sim 41 \%$. Narayan et al(Narayan, 2002, PP. 149-156) applied ultrasonic technology to increase permeate and distillation flux of different system by $22 \% \sim 205 \%$.

\subsection{Add salt to feed solutions}

For recovering volatile solutes in VMD process, salts could be added to feed solutions to reduce water vapour pressure and thus enhance the permeation flux of volatile components. For instance, Tang et al(Tang, 2002, PP. 11-14) have observed that the presence of $\mathrm{AlCl}_{3}$ increased $\mathrm{HCl}$ permeation flux in the experiments of recovering $\mathrm{HCl}$ by $\mathrm{VMD}$.

\subsection{Select proper operation conditions}

Cold procedure side pressure played an critical role in membrane separation performance. With the decreasing of vacuum in cold procedure side, cold procedure side pressure augmented, and membrane flux and rejection rate all decreased. Consequently, when such pressure was larger than vapour critical pressure in membrane surface, decreasing such pressure would be beneficial to enhance membrane rejection rate and flux(Liu, 1997, PP. 65-68). Enhancing temperature could lower viscosity of fluid, reduce liquid membrane drag and thus increase mass transfer coefficient. For volatile components, with the increasing of concentration, membrane flux and rejection rate increased(Zhong, 2003, PP. 44-46); For nonvolatile components, with the increment of feed concentration, membrane flux lowered, because with the increasing of feed concentration, water saturated vapour pressure decreased, and separation process impetus lowered, which was not good for separation( $\mathrm{Du}, 2000$, PP. 14-17); Operation manner had significant impacts on flux. Wirth et al (2002, PP. 139-145) have investigated total heat and mass transfer coefficient in two operation manners in the experiment of desalination by water solutions, namely inside /out and outside/in, and speculated that outside/in manner was of more industrial production values.

\subsection{Ameliorate membrane performance}

Hydrophobicity is primary for membrane materials and microporous membrane during VMD process. Materials are more hydrophobic, performance of materials used for VMD is better; Membrane pore is another cardinal factor affecting flux and rejection rate during MD process. Increasing pore size and porosity would be beneficial to the increment of flux. Study by Li et al(2003, PP. 153-156) indicated that polyethylene membrane with larger micropore diameter and higher porosity had quite high permeation flux under the same operation condition; Membrane loading density also affected VMD performance, Zhu et al(1999, PP. 51-54) found that pressure difference between both membrane sides was large when membrane loading density was quite low, and thus such difference resulted in large permeation flux.

\section{Research prospects of VMD}

Compared to other MD processes, operation temperature of VMD process could be lower, and at the same temperature, its flux would be larger. VMD could be convenient to utilize cheap heat sources such as solar energy, geothermal energy, waste heat and so on(Mathiouslakis, 2007, PP. 346-365). Therefore, in combination with such cheap energy, VMD would have greater advantage in water treatment. For MD was a process of phase tansition, utilization of heat energy could reduce due to latent heat of vaporization. To design proper energy recovery facilities would be of great practical values in energy saving(Duan, 2005, PP. 15-16; Liu, 2008, PP. 32-37).

VMD is a new membrane separation technology, and its corresponding application research have obtained excellent achievements, which showed its promising application prospects. We believed that VMD would attain better application in water treatment and bring greater impetus for substantial development along with the development of membrane material and deep amelioration of process mechanism.

\section{References}

Bandini, S., \& Sarti, G.C. (1999). Heat and mass transport resistances in vacuum membrane distillation per drop. AIChEJ, 45 (7):58-62. 
Bart, V.B., \& Carlo, V. (2002). Distillation vs. membrane filtration: overview of Proeess evolutions in seawater desalination. Desalination,143(2):207-218.

Chen, C.X., Yu, L.X., \& Dai, T.Y. (1996). Review of new membrane and membrane process. Technology of Water Treatment, 22(6):307-313.

Drioli, E., \& Wu, Y.L. (1985). Membrane distillation: An experimental study. Desalination, 53:339-346.

Du, J., Liu, Z.H., \& Tao, C.Y., et al. (2000). Investigation on the treatment of aqueous $\mathrm{Cr}^{3+}$ solutions with PVDF microporous membranes. Chemical Research and Application, 20(3):14-17.

Duan, X.L., \& Chen, B.B. (2005). Study on the experimental of ultrasound enhancing vacuum membrane distillation. Chemical Engineer,118(7):15-16.

Lawson, K.W., \& Lloyd, D.R. (1997). Review: Membrane Distillation. J.Membr.Sci., 124:1-25.

Li, L.M., Xu, Z.K., \& Liu, Z.M., et al. (2003). Mcroporous polypropylene and polyethylene hollow fiber membranes. Part 3. Experimental studies on membrane distillation for desalination. Desalination, 155:153-156.

Li, Y.L. (2001). The Present Situation and the Application about Seawater Desalting. Water Conservancy Science and Technology and Economy, 7(4):166-167.

Lin, S.Q. (2000). The present situation and future of seawater desalination. Technology of Water Treatment, $26(1): 7-12$.

Liu, G.X., \& Liu, L.S. (1991). Membrane separation technology and its application. Beijing:Chemical Industry Press, 125.

Liu, L.Y., Ding, C.W., \& Chang, L.J., et al. (2008). Progress in membrane separation enhanced by ultrasound. Chemical Industry and Engineering Progess,27(4):32-37.

Liu, M.L., Huo, Z.X., \& Chen, D.J., et al. (1997). The influence of the degree of vacuum of the cold side on vacuum membrane distillation process. Membrane Science and Technology, 17(3) :65-68.

Martinez, D.L., Vazquez, G.M.I., \& Florido, D.F.J. (1998). Study of membrane distillation using channel spacers. $J$ Memb Sci., 144 (1-2) :45-56.

Mathiouslakis, E., Belessiotis, V., \& Delyannis, E. (2007). Desalination by using alternative energy: review and state-of-the-art. Centre National de La Recherche Scientifioue, 203(1-3):346-365.

Narayan, A.V., Nagaraj, N., \& Hebbar, H.U., et al.(2002). Acoustic field-assisted osmotic membrane distillation. Desalination, 147(1-3):149-156.

Schneider, K., \& Gassel, T.J. (1984). Membrane distillation. Chem.Ing.Tech., 56:514-521.

Tang, J.J., Zhou, K.G., \& Zhang, Q.X. (2002). $\mathrm{HCl}$ separation from $\mathrm{AlCl}_{3}-\mathrm{HCl}-\mathrm{H}_{2} \mathrm{O}$ system by vacuum membrane distillation. Membrane Science and Technology, 22 (3) :11-14.

Wirth, D., \& Cabassud, C. (2002). Water desalination using membrane distillation: comparison between inside/out and outside/in permeation. Desalination, 147(1-3):139-145.

Xu, Y.Y., Xiang, H., \& Chen, Y.S. (1998). The study and development of membrane distillation. Membrane Science and Technology, 18(6):1-6.

Zhang, Y.J., \& Wu, X.M., et al. (2001). Application and development of membrane distillation. Journal of Filtration \& Separation, 11(2):16-21.

Zhong, S.A., Li, Y.P., \& Zhou, C.S., et al. (2003). Study of vacuum membrane distillation for polyphenol pharmacy wastewater. Industrial Water Treatment, 23(4):44-46.

Zhu, B.K., Xu, Y.Y., \& Xiang, H., et al. (1999). Vacuum membrane distillation of polypropylene hollow fiber microporous membrane. Membrane Science and Technology, 19(5):51-54. 